\title{
Saber no alcanza para actuar: revisión y reflexiones acerca de la relación entre el conocimiento y la adopción de conductas ambientales
}

\author{
Knowing is not enough for action: review and reflections \\ on the relationship between knowledge and the adoption of \\ environmental behaviours
}

Elizabeth Gonzalez Urda ${ }^{1}$. Leonor Bonan ${ }^{1}$

\begin{abstract}
Resumen: La adopción de conductas ambientales es una meta de la Educación Ambiental (EA) y un objetivo explícito de numerosos diseños curriculares. Sin embargo, la bibliografía da cuenta de la dificultad de lograr esa meta. Estas evidencias cuestionan la eficacia de la EA. A partir de revisiones bibliográficas acerca de la adopción de conductas, provenientes de otras disciplinas, principalmente la Psicología y la Sociología, se propone un aporte al campo de la Didáctica de las Ciencias, - del cual la EA forma parte -, proveyendo algunos criterios para la reflexión acerca del rol y tipo de conocimiento promotor de conductas así como de las posibilidades de acción de los educadores. Estos aportes extra-campo iluminan la complejidad de variables que intervienen en la promoción de conductas ambientales cuestionando la idea "mágica", que circula entre muchos educadores y otros actores sociales, de que el conocimiento ambiental deriva espontáneamente en una acción ambiental.
\end{abstract}

Palabras clave: Educación ambiental. Conocimiento ambiental. Didáctica de las ciencias.

\begin{abstract}
The adoption of behaviours for the environment is a goal of Environmental Education (EE) and an explicit objective of many curricular designs. However, the bibliography tells us about the difficulty of achieving that goal. This evidence is questioning the effectiveness of the EE. From literature reviews about the adoption of behaviours from other disciplines, mainly Psychology and Sociology, a contribution to the field of the Didactics of Science, - of which the EE is a part - is proposed, providing some criteria for reflection about the role and type of promoter knowledge of behaviour as well as possibilities of action for educators. These extra - field contributions illuminate the complexity of variables that are involved in the promotion of environmental behavior, questioning the "magic" idea, -which circulates among many educators and other social actors-, that knowledge about environmental issues derives spontaneously within environmental actions.
\end{abstract}

Keywords: Environmental education. Environmental knowledge. Didactics of Science.

\footnotetext{
${ }^{1}$ Universidad de Buenos Aires, Instituto de Investigaciones CeFIEC, Facultad de Ciencias Exactas y Naturales, Buenos Aires, Argentina. E-mail: <eliurda@gmail.com>.
} 


\section{Introducción}

En Argentina, hay una gran cantidad de experiencias de Educación Ambiental (en adelante EA), pero poca evaluación de la misma más allá de los resultados escolares. González Urda, Foguelman y Zerboni (2010) señalan que la mayor parte de las recopilaciones de trabajos presentados en congresos y encuentros realizados en la Argentina en los últimos 15 años, refieren que las experiencias lograron la concientización y el entusiasmo de los estudiantes y/o la comunidad, pero pocas de ellas dan cuenta del logro, perdurabilidad y extensión social de los cambios de conducta. Por lo tanto, puede suponerse que a la fecha, la EA argentina estaría focalizada en el conocimiento y en la concientización.

Sin embargo, la EA en tanto educación científica, tiene tres finalidades generales con niveles crecientes de complejidad cognitiva: (a) la apropiación del conocimiento (conocer); (b) la relación entre conocimientos (comprender); (c) la aplicación de conocimientos (transferir a diferentes situaciones, entre ellas, las que suponen la acción). Los documentos de los encuentros internacionales de la UNESCO $(1975,1978)$ que fueron y son la base de los desarrollos curriculares de numerosos países, entre ellos la Argentina, recomiendan que la EA dispense conocimientos y fomente valores que favorezcan el desarrollo de comportamientos compatibles con la preservación del medio, así como competencias para la concepción y aplicación de soluciones eficaces a los problemas ambientales.

Este artículo nace del cuestionamiento personal como educadoras ambientales y de la necesidad de índole pragmática de evaluar la eficacia de nuestras acciones educativas como generadoras de conductas. Se basa en revisiones bibliográficas y otras publicaciones que abordan la problemática de la adopción de conductas, en algunos casos ambientales, desde el enfoque de otras disciplinas principalmente la Psicología y la Sociología. Pretende ser un aporte al campo de la Didáctica de las Ciencias, del cual la EA forma parte, iluminando la relación entre los conocimientos y la adopción de conductas ambientales, identificando y describiendo las principales líneas de investigación que refieren directa o indirectamente a esta temática y proveyendo algunos criterios para la reflexión acerca de las acciones de educación científica, especialmente las de índole ambiental, y su evaluación.

\section{Líneas de investigación acerca de la relación entre los conocimientos y las conductas}

Según González Urda (2012), en la bibliografía científica las conductas ambientales reciben muy diversas denominaciones. A los fines de este trabajo consideraremos como acciones o conductas ambientales a aquellas conductas concientes que realizan los sujetos con la intención de favorecer la relación armónica entre los sistemas sociales y naturales.

Desde mediados del siglo XX, la Psicología y la Sociología, entre otras disciplinas, enriquecieron la comprensión de la relación entre el conocimiento y la adopción de conductas, cuestión que es de sumo interés para los docentes y especialistas en Didáctica de las Ciencias Naturales, especialmente para los educadores ambientales. Como toda disciplina reciente, la Didáctica de las Ciencias Naturales ha abrevado en otras que, actuando como fuentes, han aportado marcos conceptuales y metodológicos para la descripción y comprensión de los problemas de investigación y 
[...] auxilian el estudio de aspectos que no cuentan con antecedentes propios en el ámbito de la Didáctica de las Ciencias Naturales. Estos recursos darán lugar a rastrear los modelos teóricos y las estrategias metodológicas que guíen la definición y el desarrollo de la investigación. (BONAN, 2007, p. 125).

Tomando como base los trabajos de Corral-Verdugo y Pinheiro (2004), González López (2002), Kollmuss y Agyeman (2002) y Ortega (2007) se identificaron tres líneas de abordaje de la temática que, si bien surgieron sucesivamente, se mantienen vigentes en la producción científica y en muchos casos subyacen al amplio abanico de acciones de EA que promueven la adopción de conductas ambientales. Estas líneas de investigación pueden agruparse según los modelos ${ }^{2}$ que las sustentan en: (a) líneas psicológicas; (b) líneas que focalizando en los individuos relacionan variables psicológicas y de contexto; y (c) líneas sociológicas. Para ilustrar cada línea destacaremos a algunos de los autores que son referentes de las mismas y/o cuyos aportes resultan relevantes para nuestra discusión.

\section{Líneas psicológicas}

Sobre finales de la década de 1960, algunos modelos de la Psicología caracterizaron las relaciones entre el conocimiento del individuo y la adopción de conductas. Estas primeras líneas de investigación partieron de un Modelo inicial (Figura 1) en el que, según Fishbein y Ajzen (2011), se asume que el ser humano es un ser racional cuyas conductas sociales derivan de la información o creencias que posee acerca de una determinada cuestión de manera razonable y usualmente espontánea. Desde esta perspectiva la relación entre el conocimiento y la acción o conducta está mediada por actitudes tales como la conciencia y la preocupación.

Figura 1. Modelo inicial de relación entre el conocimiento y la conducta

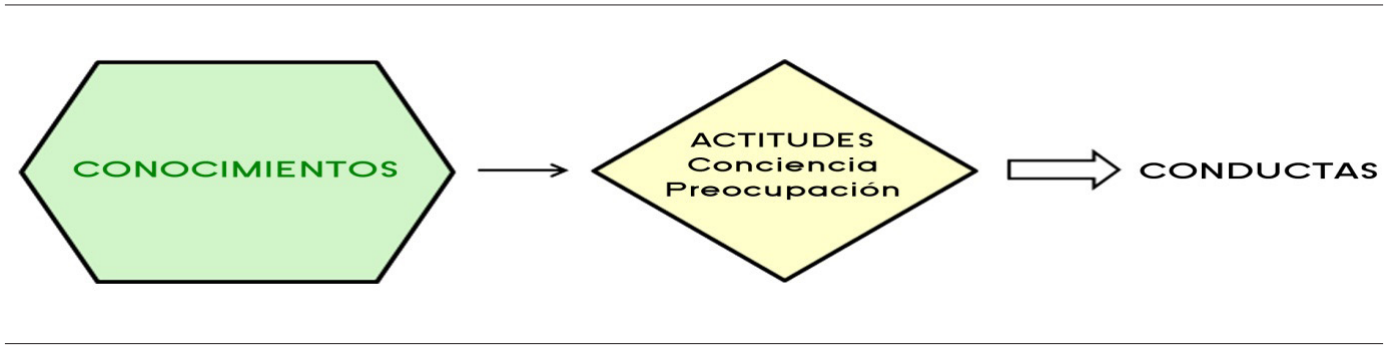

Fuente: Kollmuss y Agyeman (2002).

\footnotetext{
${ }^{2}$ Asumiremos la noción de modelo teórico de Giere (1992) que ha adquirido gran consenso en la producción teórica de Didáctica de las Ciencias Naturales. Esta asume que un modelo es un ente abstracto creado por un científico para representar la porción del mundo que pretende explicar a través de sus teorizaciones. Este modelo posee una relación de semejanza en ciertos aspectos y en ciertos grados con el objeto del mundo a representar.
} 
A lo largo de las décadas siguientes, las investigaciones de esta línea se enfocaron en la identificación y manipulación de variables de carácter individual, típicas de los estudios psicológicos (actitudes, creencias, valores, normas personales), en busca de relaciones causales entre ellas y la consecuente predictibilidad de las conductas. Los diferentes autores enriquecieron o modificaron las interacciones entre las variables con respecto al modelo inicial. En la actualidad pueden diferenciarse varias orientaciones dentro de esta línea psicológica tales como la orientación conductual o la prosocial, entre otras.

Fishbein y Ajzen (2011), referentes de la línea conductual, con su "Teoría de la acción razonada" y con la "Teoría de la conducta planificada", elaboraron, entre 1975 y 1980, modelos más complejos en los que incorporan a las intenciones como variable que dispone y permite predecir las acciones (Figura 2). Centrados en el individuo, diferencian la información (factor contextual) de las creencias (variable interna). Estas últimas se organizan en función de la personalidad, las características socioeconómicas y la información de la que dispone el individuo, que incluye su conocimiento. Según estos autores, la educación formal, los medios de comunicación y la familia entre otros, son fuentes de la información, modelan las creencias y condicionan las conductas que adoptan los sujetos.

Figura 2. Modelo de Fishbein y Ajzen

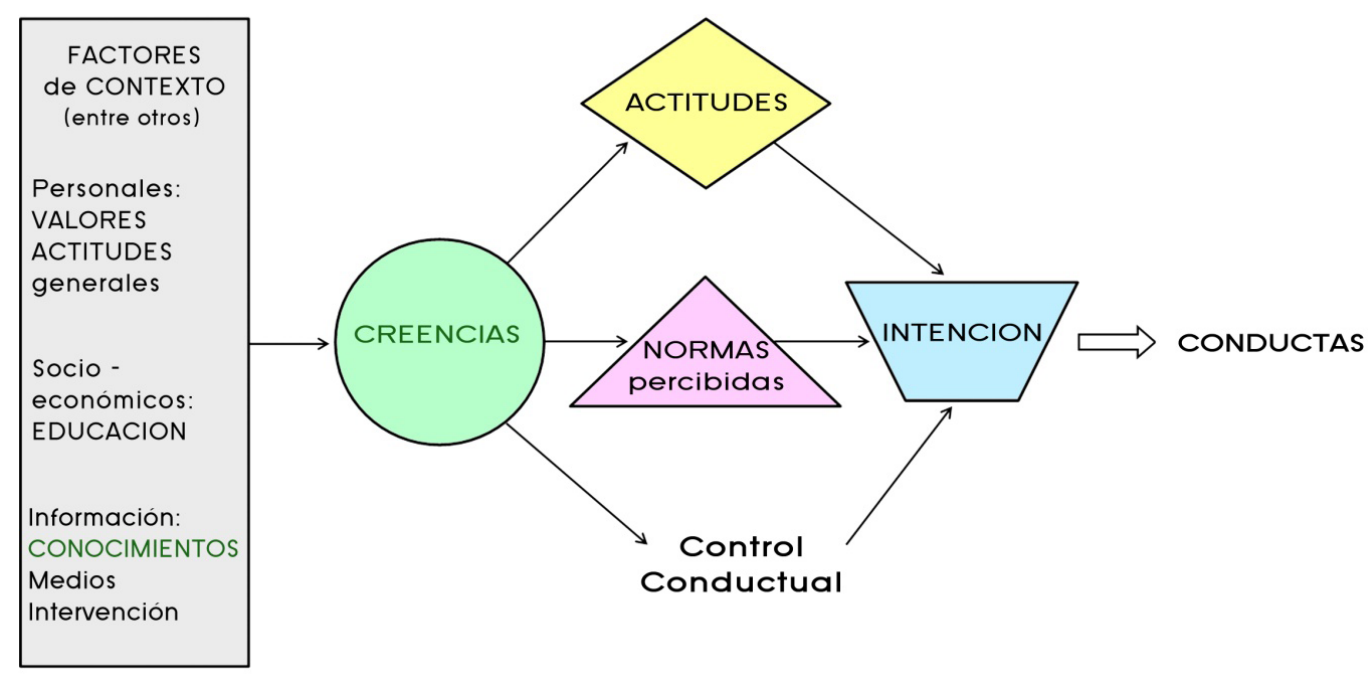

Fuente: Adaptado de Fishbein y Ajzen (2011).

Basados en la Teoría de la conducta planificada, Hines, Hungerford y Tomera (1987) propusieron el Modelo de conducta ambiental responsable. Identificaron variables asociadas a este tipo específico de conductas, como por ejemplo el sentido de responsabilidad individual, el compromiso verbal ante otros sujetos y la expectativa individual acerca de tener éxito o recibir un refuerzo 
positivo al llevar adelante una conducta (locus de control). En esta propuesta el conocimiento es un prerrequisito para la adopción de una conducta ambiental responsable. Estos autores, diferencian entre el conocimiento del tema (información) y el conocimiento y las habilidades personales para la acción que, dada una situación aumentan la efectividad de la acción. Asimismo diferencian la información de la comprensión del tema (conocimiento profundo de la naturaleza del asunto y sus implicaciones naturales y humanas). Incluyen en su análisis, aunque no desarrollan, la influencia de los factores situacionales (restricciones económicas, presiones sociales, oportunidades de elección) que pueden tanto promover como dificultar las conductas (Figura 3).

Figura 3. Modelo de Hines, Hungerford y Tomera (1987)

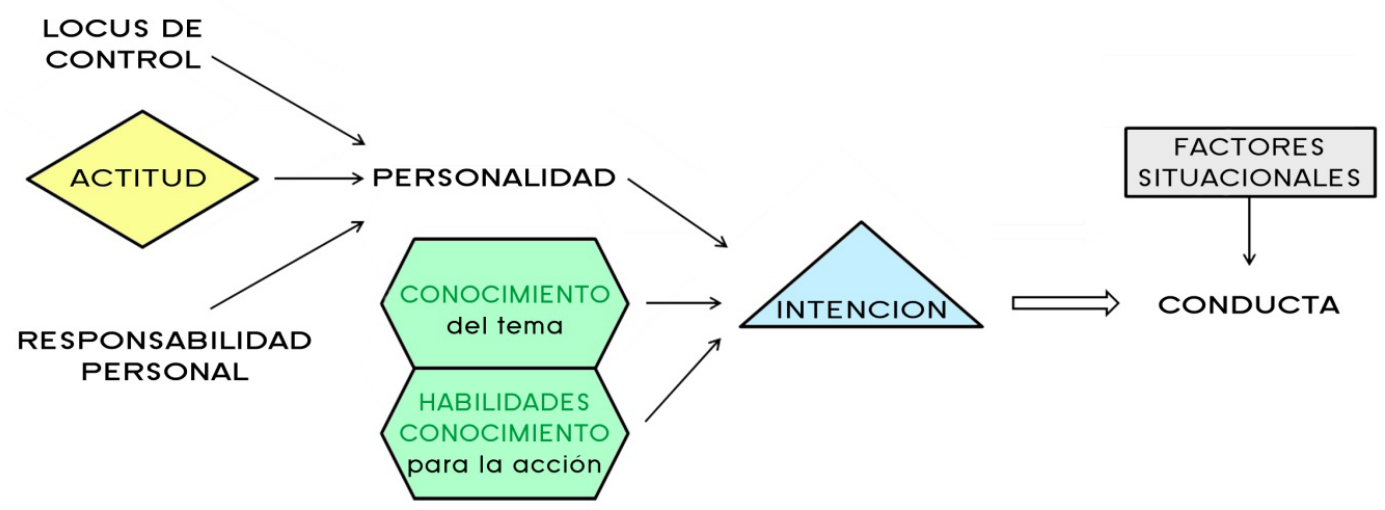

Fuente: Adaptado de Hungerford y Volk (1990).

Otra de las líneas de investigación psicológica centra su interés en la relación entre las conductas y los valores caracterizando las conductas ambientales como un tipo de conducta prosocial. Éstas suponen una descentración de los individuos de sus propios intereses para priorizar los intereses comunes o el bienestar de otras especies. Son conductas intencionales y costosas para el individuo en términos de dinero o tiempo que, aunque puedan ofrecerles poco beneficio directo, le reportan beneficios emocionales. Por ejemplo, Geller (1995) desarrolló la Hipótesis de los cuidados activos basándose en el altruismo según la cual el individuo, una vez satisfechas algunas de sus propias necesidades, puede dirigir sus conductas a favor de los otros o de la naturaleza. Geller conecta la Psicología basada en las características personales con el conductismo, al que considera la herramienta para cambiar conductas y actitudes.

\section{Líneas de investigación que relacionan variables personales y sociales}

Se incluyen en este grupo las investigaciones que, basadas en los individuos, relacionan tanto variables psicológicas como sociales ya sea en su carácter contextual o como configuradoras 
de la conducta. Algunas de estas líneas sostienen abordajes psicométricos y predictivos, característicos de los modelos anteriores y en otras se incorporan métodos y/o criterios cualitativos.

A partir de mediados de la década de 1980, Stern y colaboradores profundizaron el estudio de las relaciones entre los valores y las conductas y plantearon una sucesión de diferentes modelos que explican las conductas sociales y ambientales en particular. A lo largo de sus investigaciones analizan la relación de las variables personales y las variables contextuales a través del estudio de los procesos de interacción en los que participan tanto los seres humanos como sus construcciones sociales y contextuales. En 1999 formularon la Teoría Valor-Creencia-Norma del Ambientalismo (Value-Belief-Norm, VBN) (STERN, 2000; STERN et al., 1999). Para este grupo de investigación, la información sobre los problemas ambientales hace que se los considere no sólo como una situación desafortunada sino como algo moralmente intolerable de manera tal que esa disonancia desencadena una conducta. La Teoría VBN propone una cadena de relaciones que partiendo de los valores personales (biosféricos, altruistas, egoístas) genera diferentes tipos de conductas ambientales tanto en la esfera pública como la privada (ambientalismo, activismo, etc.). En esta teoría, los valores y las creencias son los filtros de toda nueva información o idea, de manera tal que si la información es congruente con los valores es más probable que influya sobre las creencias y actitudes, y pueda generar o modificar conductas (Figura 4).

Figura 4. Teoría Valores-Creencias-Normas del Ambientalismo (Value-Belief-Norm, VBN)

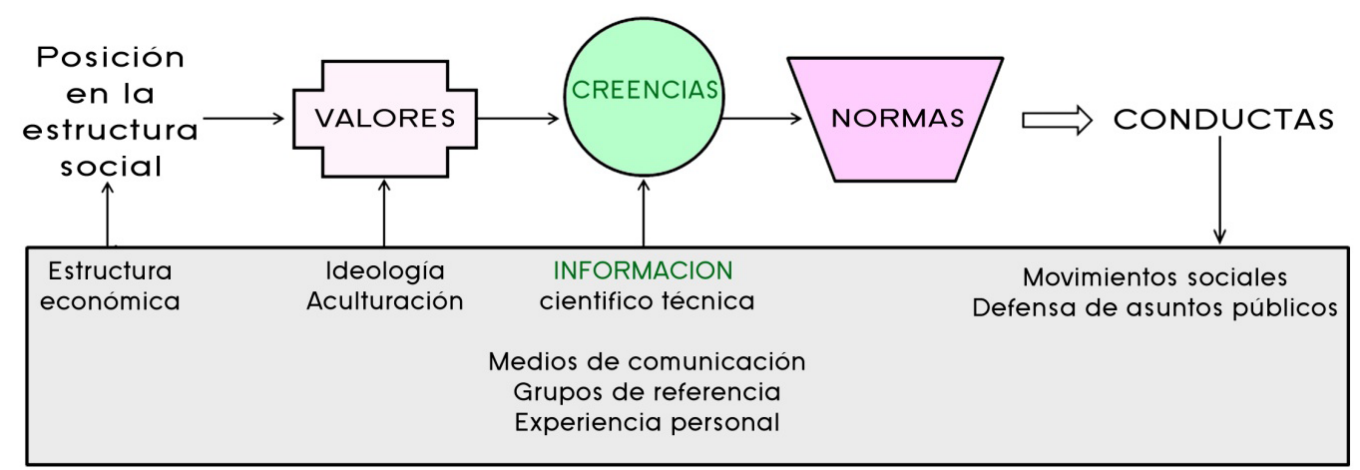

Fuente: Adaptado de Stern (2000) y Stern et al. (1999).

González López (2002) propone un Modelo Cognitivo de Conducta Ecológica e identifica los procesos cognitivos asociados con los valores y tendencias personales definidos por Stern et al. (1999). Para González López, la negación, minimización o aceptación del deterioro ambiental como inevitable es un mecanismo cognitivo que obstaculiza la acción mientras que el reconocimiento de los problemas ambientales y la predisposición a pensar en nuevos términos son los que tienen mayor relación con las conductas ambientales. 
Otro modelo focalizado en los aspectos cognitivos que considera variables sociales es el de Wagner (2003). Este autor critica fuertemente la fragmentación de los estudios psicológicos que aceptan acríticamente el principio heurístico de la causalidad, característico de las Ciencias Naturales. Estudia las conductas de los consumidores con un doble abordaje: el cognitivo psicológico y el cognitivo antropológico. Según Wagner (2003), dado que la Psicología cognitiva toma a la cognición como el procesamiento de la información y la Antropología la aborda desde la perspectiva de la resolución de problemas en un determinado contexto, este tipo de abordaje amplía la escala de estudio, cambia la unidad de análisis y permite una mejor comprensión de las conductas en situaciones reales. Toma como base la Teoría de la Acción Razonada e incorpora conceptos antropológicos, tales como el de pensamiento práctico y bricolage propuesto por Lévi-Strauss (1966), a los aspectos cognitivos. A diferencia del Modelo inicial, los factores cognitivos tendrían una influencia directa sobre la adopción de conductas regulando la intención de actuar, mientras que los factores motivacionales entre los que se encuentran los valores, las actitudes y los deseos, la predisponen (Figura 5).

Figura 5. Modelo cognitivo de Wagner (2003)

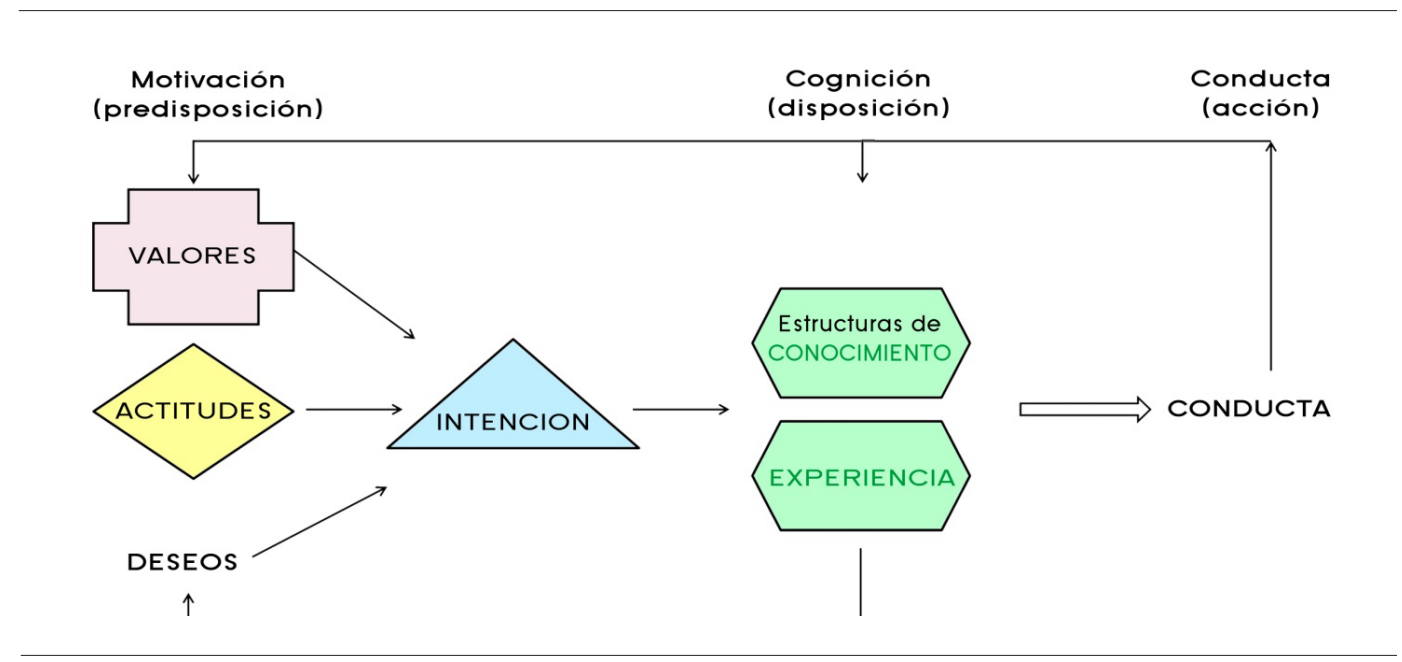

Fuente: Adaptado de Wagner (2003).

Kollmuss y Agyeman (2002), elaboran un modelo en el que destacan la importancia de los viejos hábitos personales como muy fuertes barreras de la acción ambiental. Incluyen al conocimiento, los valores, las actitudes y el involucramiento emocional en un complejo que denominan "conciencia ambiental" de manera tal que para estos autores no hay una relación directa entre conocimiento y conducta ambiental. Los distintos componentes del modelo se mantendrían a lo largo de la vida del individuo aunque la importancia relativa de cada uno de 
ellos variaría. Si bien incorporan variables sociales, proponen a la práctica repetitiva como estrategia para la generación de nuevas conductas.

En cambio para González López (2002) la identificación, comprensión y transformación de los factores sociales, situacionales y contextuales son la clave de un cambio efectivo de las conductas ambientales por su impacto sobre las mismas y su influencia sobre los factores de tipo personal. Con más énfasis Berenguer y Corraliza (2000) señalan que los factores de control y de preocupación social son los predictores conductuales más consistentes.

\section{Líneas de investigación basadas en modelos sociológicos}

En esta categoría el objeto de estudio no es el individuo sino un grupo social, grandes colectivos y/o instituciones u organizaciones sociales, por ejemplo los ciudadanos, los consumidores, el público, el barrio, la ciudad, el país, la empresa u otra organización. Muchas de estas investigaciones están asociadas con el marketing, las políticas públicas, la toma de decisiones grupales o la comunicación masiva. Analizan cómo los individuos se comportan en un grupo o cómo y cuáles son los mecanismos sociales que influyen sobre las decisiones individuales de grandes grupos de personas u organizaciones. El abordaje de la diversidad de variables que subsumen estos estudios supera el objetivo de esta presentación.

Como ejemplo se muestra en la Figura 6, la propuesta de Blake (1999) quien profundiza en el estudio de la relación valores-conducta con respecto a la aplicación de políticas ambientales. Identifica las barreras individuales y sociales que se establecen entre la preocupación ambiental y la acción. Modela un continuo desde las características individuales hasta las variables sociales que pueden entorpecer la adopción de conductas ambientales (Figura 6).

Figura 6. Modelo de Blake (1999)
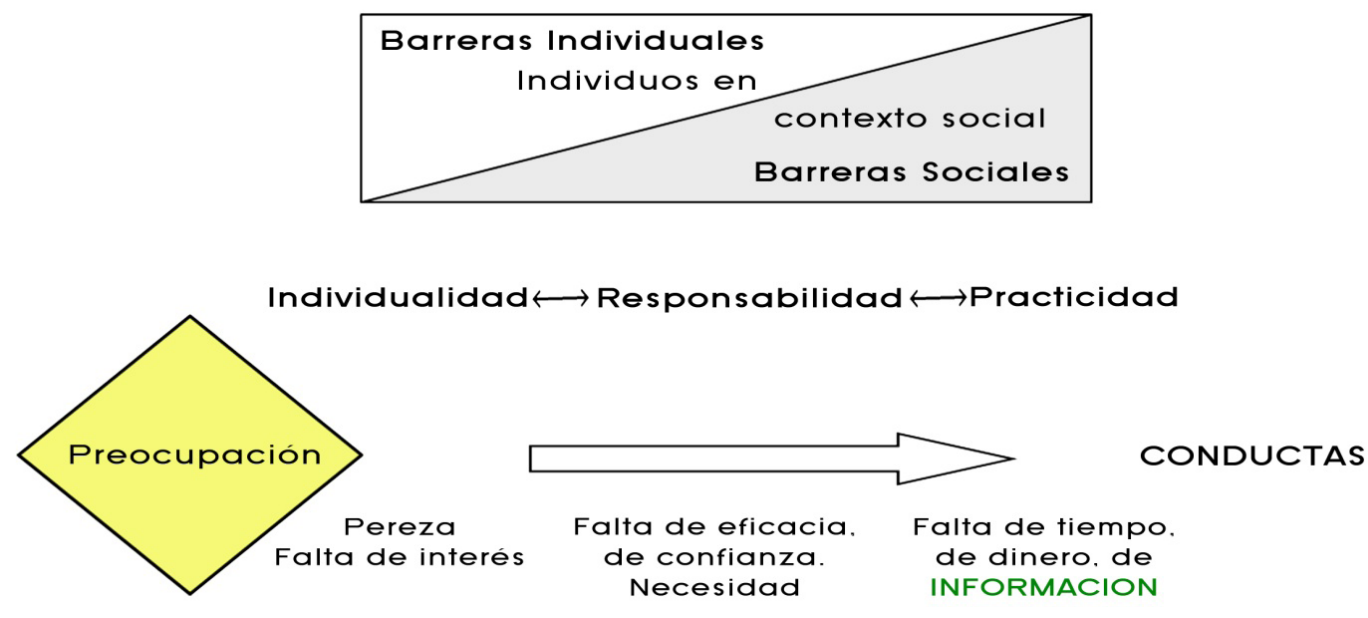

Fuente: Adaptado de Kollmuss y Agyeman (2002). 
En este grupo también pueden destacarse los trabajos acerca de los procedimientos deliberativos e inclusivos (deliberative and inclusory proceedures, DIPS) y el marketing social comunitario para la sustentabilidad (community social marketing for sustainability). Se tratan de líneas de investigación-acción con el objetivo de resolver conflictos, generar cambios a través de la toma de decisiones, definir prioridades y encarar la gestión institucional a través de la participación de los distintos actores sociales involucrados. Con un fin pragmático, proponen un abordaje pluralista para comprender los conocimientos y valores de cada grupo y promulgan mayor participación e inclusión en la toma de decisiones y modificaciones institucionales (BROWN, 2003; DE MARCHI; RAVETZ, 2001; MCKENZIE-MOHR, 2011).

\section{Algunas reflexiones en torno a las relaciones entre los modelos y la Educación Ambiental}

Los modelos característicos de las diferentes líneas de investigación abordados en este trabajo dan cuenta de la multiplicidad de factores que influyen en la adopción de conductas, en especial las ambientales. Las investigaciones presentadas ofrecen pistas para profundizar el análisis del impacto de la EA, particularmente en relación con la adopción de conductas ambientales.

Se observa una complejización creciente del modelo inicial debido a la mayor identificación de variables internas y externas al individuo, y de diferentes relaciones entre ellas y con la adopción de conductas. También se ha pasado de la focalización en los individuos hacia la participación ciudadana y la gestión social. Se mantienen a la fecha varias líneas cuyo objeto de indagación es, en general, una pequeña parte de un todo complejo que puede, a su vez, ser abordado desde marcos teóricos diferentes. Acordamos con Kollmuss y Agyeman (2002) cuando destacan que la multiplicidad de modelos coexistentes indica que la pregunta acerca de lo que modela las conductas ambientales es tan compleja que no puede ser visualizada en un solo marco o diagrama. Ese utópico diagrama podría ser tan complicado que perdería su practicidad y probablemente hasta su significado.

Por otra parte, se partió de una concepción indiferenciada de conocimiento llegando a apelar a diferentes conceptos que si bien no son sinónimos, dan cuenta o se relacionan con algún aspecto de la cognición: información, creencias, conocimiento (este último bajo las formas de "contenidos y operaciones cognitivas", "conocimiento del tema" y "conocimiento y habilidad para la acción” o "conocimiento científico o social”), etc. Teniendo presente esta aclaración se observa que, a excepción del modelo de Wagner (2003) en el que conocimiento tiene una influencia directa sobre la adopción de conductas, en general puede afirmarse que el conocimiento es condición necesaria para la adopción de conductas, pero no es la condición más importante ni tampoco determinante.

La bibliografía señala que las relaciones directas y lineales se mostraron insuficientes para explicar y/o predecir las conductas ambientales, dando cuenta de brechas entre las conductas y los valores, las actitudes, la conciencia o el conocimiento (BLAKE, 1999; KOLLMUSS; AGYEMAN, 2002). Es decir, ninguna de estas variables típicas de los modelos netamente psicológicos se muestra como determinante de la actuación ambiental, tampoco pueden establecerse relaciones causales y mucho menos generalizables y previsibles entre las diferentes variables psicológicas de manera tal que los factores sociales, culturales, coyunturales o la temática específica son para 
algunos autores los más importantes. En oposición a la mayor parte de la literatura, Fishbein y Ajzen (2011) consideran que su Teoría de la acción razonada es un marco conceptual unificador que puede ser aplicado para predecir y entender cualquier conducta humana.

Desde nuestro punto de vista, la consideración del contexto no sólo es ineludible para la comprensión de la adopción de conductas ambientales, sino que las variables psicológicas son también producto de la interacción del individuo con su entorno. Si bien acordamos con el hecho de que las variables enumeradas aportan a la comprensión del fenómeno de las conductas ambientales, estos modelos, al estar situados en el individuo y con este abordaje metodológico, recortan y dejan fuera de análisis al contexto histórico social, fuerte determinante de cualquier situación ambiental y desde luego, de las conductas asociadas a ella.

No obstante lo señalado, el Modelo de conducta ambiental responsable de Hines, Hungerford y Tomera (1987) fue muy influyente en educación ya que además de ser pionero en el estudio de la adopción de conductas ambientales es consonante con las finalidades y recomendaciones de la UNESCO cuyos documentos técnicos - entre ellos, Benet (1991) - fueron ampliamente difundidos y replicados en los desarrollos curriculares de numerosos países. Las propuestas didácticas derivadas, por ejemplo, Hungerford y Volk (1990) focalizan en las acciones individuales con implicaciones personales y/o sociales, pero no promueven el cuestionamiento del sistema socioeconómico, importante condicionante de los problemas ambientales.

En cuanto a las líneas de investigación netamente sociales, - si bien algunas de ellas tienen una clara orientación hacia el marketing, el consumo, la información y las políticas públicas -, podrían brindar herramientas y procedimientos para la EA a gran escala o en el caso de las corrientes de EA que promueven la participación social, previo análisis crítico de sus marcos filosófico-políticos.

\section{Reflexiones para la Educación Ambiental}

Los trabajos de Hungerford y Volk (1990) o el modelo de Wagner (2003) podrían ser útiles a la EA ya que apelan a conceptos que figuran habitualmente en los diseños curriculares. Sin embargo, las investigaciones acerca de la relación entre el conocimiento y las conductas ambientales dejan en claro la existencia de limitaciones. Surge de manera contundente que el conocimiento, y particularmente el conocimiento escolar, no basta para generar acciones ambientalmente comprometidas. Es necesaria la concurrencia de otros factores, muchos de los cuales no dependen de la enseñanza formal. Abundan los ejemplos en que las conductas ambientales se logran porque son convenientes para el sujeto en términos económicos o, en el ámbito educativo, ante la necesidad de aprobar una evaluación. En otros casos los medios de comunicación o las políticas socioeconómicas tienen mayor efecto sobre las conductas que una acción educativa. Es decir, para actuar en algunas situaciones ni siquiera es necesario conocer el tema en profundidad. No obstante, creemos que cuando la adopción de una conducta está dada por la primacía de situaciones coyunturales y no por la comprensión de su efecto e importancia, es de esperar que la misma sea más susceptible a los cambios del entorno y se sostenga en tanto se mantengan los estímulos externos, como por ejemplo, el precio de un producto o la presión de una evaluación. No es ésta la EA a la que aspiramos. La comprensión integral de la temática ambiental permite fundamentar las decisiones, las conductas y su mantenimiento 
temporal, es decir puede aportarle significatividad a los contenidos, favorecer el involucramiento y la adopción de conductas. Estas afirmaciones están en línea con la noción de alfabetización científico-tecnológica (FOUREZ, 2005), una línea de investigación muy fructífera en la Didáctica de las Ciencias Naturales que amplía los horizontes de la enseñanza de estas ciencias integrando enfoques sociales.

Nuestra realidad nos muestra que cuanto más significativo es un contenido, cuanto más involucrados estamos con él, mayor será la intención de actuar, pero para actuar hace falta algo más: creer que podemos actuar y que lo que hagamos tendrá algún resultado favorable. Aún en el caso de cuestiones globales habrá alternativas de abordaje y acción local tanto a escala social como personal. Una decisión didáctica puede ser explicitar esas conexiones entre el problema global y su incidencia u origen local como en el caso del cambio climático o, viceversa, entre un problema local originado por una situación global como cuando se implantan industrias sucias en países económicamente menos desarrollados para proveer insumos "limpios" a los más desarrollados. Ahora bien, la bibliografía muestra que con conocer o comprender no se asegura la adopción de conductas. La complejidad del ambiente tanto en términos de escala (local-global) y variables (naturales-sociales) como la complejidad de la adopción de conductas fundadas y duraderas permiten pensar y enseñar la temática ambiental desde una perspectiva más integral y menos ingenua. Otra decisión son las estrategias didácticas que se apliquen. La EA basada en el análisis de casos, el aprendizaje basado en problemas, el diseño de proyectos, las prácticas profesionalizantes pueden incorporar las variables sociales, darle significatividad al contenido, propiciar el involucramiento y promover la acción ya que pueden darle al estudiante no sólo conocimientos complejos sino también confianza para actuar fuera de las aulas. Este tipo de estrategias ponen en juego variados tipos de conocimiento y pueden enfrentar al estudiante a situaciones de práctica en contexto dándole confianza para actuar y conciencia de sus posibilidades y los alcances de su acción.

No obstante, la adopción de conductas no sólo requiere de los conocimientos y habilidades desarrollados en ámbitos educativos sino también de los hechos y acciones que se promuevan en ámbitos e instituciones extraescolares. La adopción de conductas será mucho menos alcanzable si el análisis se limita a pensar sólo en lo que puede hacerse desde las instituciones educativas y a nivel individual.

Es la propia complejidad de la generación y/o adopción de conductas la que relativiza el cuestionamiento hacia la efectividad de la EA. En todas las sociedades, los actores sociales que tienen responsabilidad en la adopción de conductas ambientales son varios y diversos, entre ellos, los poderes ejecutivos y legislativos de las distintas jurisdicciones políticas, los medios de difusión e información y, desde luego, los educadores.

La precedente revisión bibliográfica nos permitió reflexionar sobre cuestiones generales de la EA y generó algunos interrogantes que nos interpelan como educadores:

1. ¿Cuál es el rol del conocimiento escolar en la adopción de conductas ambientales?

En primer lugar, nos referimos al conocimiento escolar en relación con los ámbitos de educación formal en cualquiera de sus niveles. Acordamos con Kollmuss y Agyeman (2002), cuando consideran que cuanto más prolongada es la educación, los conocimientos acerca de los temas ambientales podrán ser más extensos, pero no necesariamente aumentará la actividad ambiental. Independientemente del nivel educativo del que se trate, la influencia de la escolaridad en la adopción de conductas es variable y muchos autores han planteado las dificultades 
en la enseñanza de conductas ambientales en ámbitos educativos formales (CHAWLA, 1999; CULEN; VOLK, 2000; HUNGERFORD; VOLK, 1990; KOLLMUSS; AGYEMAN, 2002; NIETO CARAVEO, 2004).

Con esta revisión vemos que el rol del conocimiento escolar en la adopción de conductas ambientales que perduren en el tiempo es importante aunque tiene sus limitaciones pues al estar involucrados otros actores y conocimientos sociales se diluye su potencia. Para ampliar la efectividad de la EA, es necesario, por un lado, incorporar en la enseñanza los variados componentes de la cuestión ambiental (variables naturales-sociales, enfoque global-local) y de la didáctica (selección de contenidos de ciencias naturales a la luz de la alfabetización científica, y con ello, la inclusión de enfoques provenientes de las ciencias sociales para realizar dicha selección; resolución de problemas como actividades de desarrollo de los contenidos; autorregulación como modo de evaluación del aprendizaje, incluyendo la identificación de actitudes frente a las problemáticas ambientales y su evolución en el tiempo; visibilizar la dialéctica que se establece entre la teoría y la práctica, entre los factores de mayor relevancia) y, por otro, la acción sinérgica de grupos e instituciones del contexto social de la institución educativa.

2. ¿Cuál es el conocimiento promotor de conductas ambientales?

Profusa bibliografía da cuenta de que las representaciones del ambiente que tienen los estudiantes, los docentes y la población en general son estereotipadas, marcadamente naturalistas excluyendo o minimizando los componentes sociales del concepto (FLORES, 2008, 2010; GONZÁLEZ URDA, 2004, 2010; MOLFI GOYA, 2000; SILVA, 2002). Estas concepciones podrían ser una explicación de la escasa alusión a las conductas ambientales ya referida en la introducción de este trabajo. Por lo tanto y como primer paso, consideramos conveniente explicitar y revisar las propias concepciones de los educadores ambientales. También, la EA no sólo debería incluir los conceptos ambientales sino también las herramientas para la actuación tanto en la esfera privada como en la pública, es decir, saber y saber hacer. Por ejemplo, enseñar a separar residuos en origen sin preguntarnos qué pasa con ellos cuando el camión recolector se los lleva, puede tener un efecto adverso al buscado al generar desánimo por la falta de efectividad real de la propia acción. Por ello, incluir otros contenidos tales como los ciclos de vida de los productos, los procesos de reclamo o elevación de propuestas a las administraciones locales, las vías de participación ciudadana, entre otros, no sólo amplía la escala de análisis del problema y extiende la responsabilidad de su solución desde el individuo a las instituciones socioeconómicas y políticas responsables, sino que podrían generar la confianza suficiente para que el estudiante actúe.

Esta revisión muestra también que es ingenuo asumir, a la manera de las líneas psicológicas, que el ser humano hace un uso sistémico de la información y que tiene valores permanentes. Es habitual que en los conflictos ambientales se observen dificultades de diálogo y consenso entre especialistas, técnicos y legos. Muchas veces, los poderes económicos y políticos y/o el miedo de la población, priman sobre los aportes del conocimiento técnico. También, sucede que cada colectivo social no tiene un discurso unificado. Estos hechos aportan un tema de reflexión adicional para los educadores ambientales: resulta indispensable la integración de abordajes provenientes de las ciencias naturales y de las sociales para la comprensión del problema ambiental y el fomento el diálogo. Siendo el conocimiento ambiental complejo, la confluencia de enfoques y actores también debe ser compleja.

3. Entonces... ¿qué pueden hacer los educadores ambientales?

Acordamos con Cuello Gijón (2003, p. 14) cuando dice: 
Es conveniente diferenciar aquellos problemas o aspectos del problema sobre los que podemos intervenir desde la acción escolar en clave de solución, de aquellos otros que por sus características (complejidad, lejanía, dimensiones, etc.) sirvan como recursos para acceder a conocimientos, afianzar valores, reforzar voluntades, etc. más que como objetivos de intervención real y directa.

Así como no se puede enseñar todo, no se puede actuar sobre todo lo que pasa. Es necesario tomar decisiones fundamentadas para seleccionar contenidos, procedimientos y también objetivos. Objetivos acordes a nuestra propia capacidad de acción como educadores de ámbitos formales. Lograr la adopción de conductas depende de la simultaneidad de diversas responsabilidades. Tal vez allí estribe el origen de lo que en algunos ámbitos se considera como una baja efectividad de la EA. Sin dudas podemos mejorar nuestra tarea, pero la finalidad de la adopción de conductas debería ser compartida por otras instancias de la comunidad.

\section{Conclusiones}

El análisis de las investigaciones y conocimientos del ámbito de la Psicología y de la Sociología cuestiona y enriquece posibles abordajes que circulan en el ámbito de la EA. Estas disciplinas pueden considerarse fuentes de la Didáctica de las Ciencias Naturales y pueden resultar útiles al abordaje de los problemas ambientales, en especial, al trascender el reduccionismo imperante en la EA. La complejidad de variables que intervienen en la promoción de conductas ambientales da cuenta de la necesidad de superar abordajes que limitan la EA al análisis de aspectos técnicos o sociales exclusivamente.

Muchos educadores ambientales apuestan al conocimiento y a través de él lograr cambios de conductas, sin embargo y por lo dicho hasta aquí, ésta es una apuesta ingenua. Tanto en el ámbito educativo como en el sentido común de la sociedad circula la idea "mágica" de que conociendo, sabiendo acerca de un tema, se generan conductas coherentes con ese conocimiento. Estas ideas fueron las que primero guiaron la investigación de la relación entre los conocimientos y las conductas. Pero lo que señalan las investigaciones provenientes de otros campos y lo que vemos a nuestro alrededor es que la adopción de conductas es un proceso mucho más complejo y que el rol que la EA puede desplegar para la adopción de conductas perdurables en el tiempo es importante aunque con limitaciones. Esta revisión provee información para fundamentar que la adopción de conductas científicamente cimentadas y duraderas no sólo requiere saber sobre el complejo tema ambiental sino también sobre estrategias de acción a escala individual y social.

$\mathrm{El}$ análisis de las investigaciones provenientes de disciplinas que funcionan a modo de fuentes de la Didáctica de la Ciencias, también brinda información para repensar las críticas a la efectividad de la EA, especialmente la EA en ámbitos educativos formales, con respecto a la adopción de conductas. Esta finalidad de la EA no se logra sólo por la acción de los educadores, sino que depende también de otros grupos e instituciones sociales. Reconocer y ampliar la responsabilidad hacia otros actores sociales no implica desligar a los educadores ambientales de su rol, pero tanto unos como otros no pueden obviar que saber no alcanza para actuar. 


\section{Referencias}

BENET, D. B. Evaluación de la educación ambiental en las escuelas: guía práctica para los maestros. Santiago, Chile: UNESCO, 1991. (Serie Educación ambiental, 12). Disponible en: <http://unesdoc.unesco.org/images/0006/000661/066120so.pdf>. Visitado el: 10 abr. 2015.

BERENGUER, J. M.; CORRALIZA, J. A. Preocupación ambiental y comportamientos ecológicos. Psicothema, Oviedo, v. 12, n. 3, p. 325-329, 2000. Disponible en: < http://www. psicothema.com/pdf/338.pdf>. Visitado el: 13 marzo 2017.

BLAKE, J. Overcoming the 'value-action gap' in environmental policy: tensions between national policy and local experience. Local Environment, Abingdon, v. 4, n. 3, p 257-278, 1999. Disponible en: < http://dx.doi.org/10.1080/13549839908725599>. Visitado el: 13 marzo 2017.

BONAN, L. ¿Cómo se define un problema de investigación en didáctica de las ciencias naturales? In: FIORITI, G.; MOGLIA, P. (Comp.). La formación docente y la investigación en didácticas específicas. San Martín: UNSAM, 2007. p. 125-130.

BROWN, K. Three challenges for a real people-centred conservation. Global Ecology and Biogeography, Chichester, v. 12, n. 2, p. 89-92, 2003.

CHAWLA, L. Life paths into effective environmental action. The Journal of Environmental Education, Philadelphia, v. 31, n. 1, p. 15-26, 1999.

CORRAL-VERDUGO, V.; PINHEIRO, J. Q. Aproximaciones al estudio de la conducta sustentable. Medio Ambiente y Comportamiento Humano, Madrid, v. 5, n. 1-2 p. 1-26, 2004. Disponible en: <http://www.psicothema.com/pdf/338.pdf>. Visitado el: 13 marzo 2017.

CULEN, G. R.; VOLK, T. L. Effects of an extended case study on environmental behavior and associated variables in seventh-and eighth-grade students. The Journal of Environmental Education, Philadelphia, v. 31, n. 2, p. 9-15, 2000. Disponible en: <http:// dx.doi.org/10.1080/00958960009598633>. Visitado el: 13 marzo 2017.

CUELLO GIJÓN, A. Problemas ambientales y educación ambiental en la escuela. [S.l.]: Centro Nacional de Educación Ambiental, 2003. Disponible en: <http://www. mapama.gob.es/es/ceneam/articulos-de-opinion/2003_03cuello_tcm7-53015.pdf $>$. Visitado el: 10 abr. 2015.

DE MARCHI, B.; RAVETZ, J. R. Participatory approaches to environmental policy. [Cambridge]: Cambridge Research for the Environment, 2001. (Policy research brief, 10). Disponible en: <http://www.clivespash.org/eve/PRB10-edu.pdf>. Visitado el: 10 abr. 2015.

FISHBEIN, M.; AJZEN, I. Predicting and changing behavior: the reasoned action approach. New York: Psychology Press, 2011.

FLORES, R. C. Medio ambiente y Educación ambiental: representaciones sociales de los profesores en formación. Magis: revista internacional de investigación en educación, Bogotá, v. 2, n. 4, p. 410-414, 2010. Disponible en: <http://www.redalyc.org/articulo. oa?id=281021692011 $>$. Visitado el: 13 marzo 2017. 
Saber no alcanza para actuar: revisión y reflexiones ...

FLORES, R. C. Representaciones sociales del medio ambiente. Perfiles Educativos, Mexico, v. 30, n. 120, p. 33-62, 2008. Disponible en: < http:/ /www.scielo.org.mx/scielo. php?script=sci_arttext\&pid=S0185-26982008000200003 >. Visitado el: 13 marzo 2017.

FOUREZ, J. Alfabetizar científica y técnicamente. In: Alfabetización científica y tecnológica: acerca de las finalidades de la enseñanza de las ciencias. Buenos Aires: Colihue, 2005. p 15-39.

GELLER, E. S. Actively caring for the environment: an integration of behaviourism and humanism. Environment and Behavior, Thousand Oaks, v. 27, n. 2, p. 184-195, 1995.

GIERE, R. N. La explicación de la ciencia: un acercamiento cognoscitivo. México: Consejo Nacional de Ciencia y Tecnología, 1992.

GONZÁLEZ LÓPEZ, A. La preocupación por la calidad del medio ambiente: un modelo cognitivo sobre la conducta ecológica. 2002. 216 h. Tesis (Doctorado) - Facultad de Psicología, Universidad Complutense de Madrid, 2002. Disponible en: < http://biblioteca. ucm.es/tesis/psi/ucm-t26479.pdf >. Visitado el: 10 abr. 2015.

GONZÁLEZ URDA, E. N. Ambientalización del curriculum en las carreras técnicas de nivel superior de gestión pública en la ciudad de Buenos Aires. 2010. 307 h. Tesis (Maestría en Enseñanza de Ciencias Exactas y Naturales) - Facultad de Ingeniería, Universidad Nacional del Comahue, Neuquén, 2010.

Las concepciones del medioambiente en estudiantes de nivel superior. Revista Iberoamericana de Educación, Madrid, feb. 2004. Disponible en: < http://www.rieoei. org/deloslectores/602Gonzalez.PDF>. Visitado el: 09 abr. 2015.

- Mucho se dice, poco se define: revisión de la definición de las conductas a favor del ambiente. In: JORNADAS NACIONALES, 10. y CONGRESO INTERNACIONAL DE ENSEÑANZA DE LA BIOLOGÍA, 5., 2012. Anales... Villa Giardino: Asociación de Docentes de Ciencias Biológicas de la Argentina, 2012.

GONZÁLEZ URDA, E.; FOGUELMAN, D.; ZERBONI, A. M. Los pedales de la bicicleta: la evaluación de la educación ambiental. Buenos Aires: Ed. Kaikron, 2010.

HINES, J. M.; HUNGERFORD, H. R.; TOMERA, A. N. Analysis and synthesis of research on responsible environmental behavior: a meta-analysis. The Journal of Environmental Education, Philadelphia, v. 18, n. 2, p. 1-8, 1987. Disponible en: <http://dx.doi.org/10.108 0/00958964.1987.9943482>. Visitado el: 13 marzo 2017.

HUNGERFORD, H. R.; VOLK, T. L. Changing learner behavior through environmental education. The Journal of Environmental Education, Philadelphia, v. 21, n. 3, p. 8-21, 1990. Disponible en: < http://dx.doi.org/10.1080/00958964.1990.10753743>. Visitado el: 13 marzo 2017.

KOLLMUSS, A.; AGYEMAN, J. Mind the gap: why do people act environmentally and what are the barriers to pro-environmental behavior? Environmental Education Research, Abingdon, v. 8, n. 3, p. 239- 260, 2002. Disponible en: <http://dx.doi. org/10.1080/13504620220145401>. Visitado el: 10 abr. 2015. 
LÉVI-STRAUSS, C. The savage mind. Chicago: University of Chicago Press, 1966.

MCKENZIE-MOHR, D. Fostering sustainable behaviour: an introduction to community-based social marketing. 3rd. ed. Gabriola Island: New Society Publishers, 2011.

MOLFI GOYA, E. M. Deconstrucción de las representaciones sobre el medio ambiente y educación ambiental. Tópicos en Educación Ambiental, México, v. 2, n. 4, p. 33-40, 2000. Disponible en: <http://www.anea.org.mx/Topicos/T\%204/Paginas\%2033\%20-\%2040. pdf $>$. Visitado el: 10 abr. 2015.

NIETO CARAVEO, M. C. L. Sabemos pero no actuamos: ¿cuál es el papel de la educación ambiental? Revista Universitarios, México, v. 12, n. 2, p. 56-61, 2004. Disponible en: <http:/ /ambiental.uaslp.mx/docs/LMNC-AU-0406-GAP.pdf>. Visitado el: 10 abr. 2015.

ORTEGA, J. Revisión bibliográfica analítica sobre los antecedentes motivacionales y cognitivos de la conducta proambiental en el consumidor a partir de los modelos psicológicos correlacionales y explicativos publicados en los últimos diez años (1996-2006). 2007. 246 h. Tesis. Universidad del Norte, Barranquilla, 2007. Disponible en: < http://manglar.uninorte.edu.co/bitstream/handle/10584/2119/55305492. pdf?sequence $=1>$. Visitado el: 10 abr. 2015.

SILVA, R. L. F. Representaciones sociales de medio ambiente y educación ambiental de docentes universitarios(as). Tópicos en Educación Ambiental, México, v. 4, n. 10, p. 22 36, 2002. Disponible en: <http://www.anea.org.mx/Topicos/T\%2010/Paginas\%2022-36. pdf $>$. Visitado el: 10 abr. 2015.

STERN, P. C. Toward a coherent theory of environmentally significant behavior. Journal of Social Issues, Hoboken, v. 56, n. 3, p. 407-424, 2000. Disponible en: <http://dx.doi. org/10.1111/0022-4537.00175>. Visitado el: 13 marzo 2017.

STERN, P. C. et al. A value-belief-norm theory of support for social movements: the case of environmentalism. Research in Human Ecology, Bar Harbor, v. 6, n. 2, p. 81-97, 1999. Disponible en: <http://cedar.wwu.edu/hcop_facpubs/1/>. Visitado el: 13 marzo 2017.

UNESCO. Carta de Belgrado: una estructura global para la educación ambiental. [Belgrado]: Unesco, [1975]. Disponible en: <http://unesdoc.unesco.org/ images/0001/000177/017772sb.pdf>. Visitado el: 10 abr. 2015.

Conferencia intergubernamental sobre educación ambiental: informe final. Paris, 1978. Disponible en: < http://unesdoc.unesco.org/images/0003/000327/032763sb. pdf $>$. Visitado el: 10 abr. 2015.

WAGNER, S. Understanding green consumer behaviour: a qualitative cognitive approach. New York: Routledge, 2003.

Artigo recebido em 09/07/2015. Aceito em 05/10/2016.

Dirección para contacto: Facultad de Ciencias Exactas y Naturales, CEFIEC, Intendente Güiraldes, 2160, Ciudad Universitaria, Buenos Aires 1428, Argentina. 